\title{
A NOTE ON EULER NUMBER AND POLYNOMIALS
}

\author{
LEE-CHAE JANG, SEOUNG-DONG KIM, DAL-WON PARK, \\ AND YOUNG-SOON RO
}

Received 21 September 2004; Accepted 16 October 2005

We investigate some properties of non-Archimedean integration which is defined by Kim. By using our results in this paper, we can give an answer to the problem which is introduced by I.-C. Huang and S.-Y. Huang in 1999.

Copyright (c) 2006 Lee-Chae Jang et al. This is an open access article distributed under the Creative Commons Attribution License, which permits unrestricted use, distribution, and reproduction in any medium, provided the original work is properly cited.

\section{Introduction}

Throughout this paper $\mathbb{Z}_{p}, \mathbb{Q}_{p}$, and $\mathbb{C}_{p}$ will, respectively, denote the ring of $p$-adic rational integers, the field of $p$-adic rational numbers, and the completion of algebraic closure of $\mathbb{Q}_{p}$. Let $v_{p}$ be the normalized exponential valuation of $\mathbb{C}_{p}$ with $|p|_{p}=p^{-v_{p}(p)}=p^{-1}$.

Let $p$ be a fixed prime number and let $l$ be a fixed integer with $(p, l)=1$. We set

$$
\begin{aligned}
X & =\underbrace{\lim }_{N}\left(\mathbb{Z} / l p^{N} \mathbb{Z}\right), \\
X^{*} & =\bigcup_{\substack{0<a<l p \\
(a, p)=1}}\left(a+l p \mathbb{Z}_{p}\right), \\
a+l p^{N} \mathbb{Z}_{p} & =\left\{x \in X \mid x \equiv a\left(\bmod l p^{N}\right)\right\},
\end{aligned}
$$

where $a \in \mathbb{Z}$ lies in $0 \leq a<l p^{N}$ (cf. $[3,4]$ ).

For any positive integer $N$, we set

$$
\mu_{1}\left(a+l p^{N} \mathbb{Z}_{p}\right)=\frac{1}{l p^{N}}
$$

and this can be extended to a distribution on $X($ see $[3,9])$. 
2 A note on Euler number and polynomials

This distribution yields an integral for nonnegative integer $m$ :

$$
\int_{X} x^{m} d \mu_{1}(x)=B_{m}
$$

where $B_{m}$ are called usual Bernoulli numbers (cf. [8]).

The Euler numbers $E_{m}$ are defined by the generating function in the complex number field as follows:

$$
\frac{2}{e^{t}+1}=\sum_{m=0}^{\infty} E_{m} \frac{t^{m}}{m !} \quad(|t|<\pi)
$$

where we use the technique method notation by replacing $E^{m}$ by $E_{m}(m \geq 0)$, symbollically (cf. $[3,5,7,9,10])$.

The Bernoulli numbers with order $k, B_{n}^{(k)}$, were defined by

$$
\left(\frac{t}{e^{t}-1}\right)^{k}=\sum_{n=0}^{\infty} B_{n}^{(k)} \frac{t^{n}}{n !} \quad(\text { cf. }[5,10]) .
$$

Let $u$ be algebraic in complex number field. Then Frobenius-Euler numbers were defined by

$$
\frac{1-u}{e^{t}-u}=\sum_{n=0}^{\infty} H_{n}(u) \frac{t^{n}}{n !} \quad(\text { cf. }[5])
$$

By (1.4) and (1.6), note that $H_{n}(-1)=E_{n}$.

In this paper, we will give the interesting formulae for sums of products of Euler numbers ( $=$ Frobenius-Euler numbers $)$ by using $p$-adic Euler integration which is defined in $[3,5,8-10]$. Our result is an answer to the problem which is introduced by I.-C. Huang and S.-Y. Huang in [2, page 179].

\section{Sums of products of Euler numbers}

Let $u \in \mathbb{C}_{p}$ with $\left|1-u^{f}\right|_{p} \geq 1$ for each positive integer $f$. Then the $p$-adic Euler measure was defined by

$$
E_{u}(x)=E_{u}\left(x+d p^{N} \mathbb{Z}_{p}\right)=\frac{u^{d p^{N}-x}}{1-u^{d p^{N}}}, \quad(\text { cf. }[3,5])
$$

Now, we define Euler polynomials with order $n$ by

$$
\left(\frac{u}{1-u}\right)^{m} H_{n}^{(m)}(u, x)=\underbrace{\int_{X} \cdots \int_{X}}_{m \text { times }}\left(x+x_{1}+\cdots+x_{m}\right)^{n} d E_{u}\left(x_{1}\right) \cdots d E_{u}\left(x_{m}\right)
$$


In the case $x=0$, we use the following notations:

$$
H_{n}^{(k)}(u, 0)=H_{n}^{(k)}(u), \quad H_{n}^{(1)}(u)=H_{n}(u) \quad(\text { cf. }[3,9]) .
$$

In [3], the following formula can be found:

$$
\int_{\mathbb{Z}_{p}} x^{n} d E_{u}(x)=\frac{u}{1-u} H_{n}(u)
$$

By (2.2) and (2.4), we easily see that $\lim _{k \rightarrow 1} H_{n}^{(k)}(u)=H_{n}(u)$.

For any positive integer $m, H_{n}^{(m)}(u, x)$ can be written by

$$
H_{n}^{(m)}(u, x)=\sum_{j=0}^{n}\left(\begin{array}{l}
n \\
j
\end{array}\right) x^{n-j} H_{j}^{(m)}(u)
$$

We may now mention the following formulae which are easy to prove:

$$
\left(\frac{u}{1-u}\right)^{m} H_{n}^{(m)}(u, x)=l^{n} \sum_{l_{1}, \ldots, l_{m}=0}^{l-1} \frac{u^{m l-\sum_{i=1}^{m} l_{i}}}{\left(1-u^{l}\right)^{m}} H_{n}^{(m)}\left(u^{l}, \frac{x+l_{1}+\cdots+l_{m}}{l}\right)
$$

where

$$
\sum_{l_{1}, \ldots, l_{m}=0}^{l-1}=\sum_{l_{1}=0}^{l-1} \sum_{l_{2}=0}^{l-1} \cdots \sum_{l_{m}=0}^{l-1}
$$

By using (2.2) and multinomial coefficients, We obtain the following theorem.

Theorem 2.1. For $\alpha_{1}, \alpha_{2}, \ldots, \alpha_{m} \in \mathbb{C}_{p}$ and positive integers $n, m$,

$$
H_{n}^{(m)}\left(u, \alpha_{1}+\alpha_{2}+\cdots+\alpha_{m}\right)=\sum_{\substack{i_{1}, \ldots, i_{m} \\
n=i_{1}+\cdots+i_{m}}}\left(\begin{array}{c}
n \\
i_{1}, \ldots, i_{m}
\end{array}\right) H_{i_{1}}\left(u, \alpha_{1}\right) H_{i_{2}}\left(u, \alpha_{2}\right) \cdots H_{i_{m}}\left(u, \alpha_{m}\right),
$$

where $\left(\begin{array}{c}n \\ i_{1}, \ldots, i_{m}\end{array}\right)$ is the multinomial coefficient.

Remark 2.2. The above theorem is an answer to the problem which was introduced in [2, page 179].

Remark 2.3. Note that $H_{n}(-1)=\sum_{k=0}^{n}\left(\begin{array}{c}n+1 \\ k\end{array}\right) 2^{k} B_{k}$, where $B_{k}$ are the $k$ th ordinary Bernoulli numbers.

Remark 2.4. By using Volkenborn integral, it was well known that

$$
\frac{t}{e^{t}-1}=\sum_{n=0}^{\infty} \int_{\mathbb{Z}_{p}} x^{n} d \mu_{1}(x) \frac{t^{n}}{n !} \quad(\text { cf. }[3,7,10]) .
$$


4 A note on Euler number and polynomials

In $[1,9]$, note that

$$
\left(\frac{t}{e^{t}-1}\right)^{k}=\sum_{n=0}^{\infty} \underbrace{\iint_{X} \cdots \int_{X}}_{k \text { times }}\left(x+x_{1}+\cdots+x_{k}\right)^{n} d \mu_{1}\left(x_{1}\right) d \mu_{1}\left(x_{2}\right) \cdots d \mu_{1}\left(x_{k}\right) \frac{t^{n}}{n !} .
$$

The Bernoulli polynomials with order $k, B_{n}^{(k)}(x)$, were defined by

$$
B_{n}^{(k)}(x)=\underbrace{\iint_{X} \cdots \int_{X}}_{k \text { times }}\left(x+x_{1}+\cdots+x_{k}\right)^{n} d \mu_{1}\left(x_{1}\right) d \mu_{1}\left(x_{2}\right) \cdots d \mu_{1}\left(x_{k}\right) \quad \text { (cf. }[7,9,10]) .
$$

In the case $x=0$, we write $B_{n}^{(k)}(0)=B_{n}^{(k)}$ (cf. [9]).

In [2], the authors proved the formulae of sums of products of Bernoulli numbers of higher order by using theory of residues. By using the properties of invariant $p$-adic integrals in this paper, we can also give the same formulae on the sums of products for $B_{n}^{(k)}$ in [2]. Let $\chi$ be a Dirichlet character with conductor $f$. We set $p^{*}=p$ for $p \geq 2$, and $p^{*}=4$ for $p=2$. Let $\bar{f}=\left(f, p^{*}\right)$ be denoted by the least common multiple of the conductor $f$ of $\chi$ and $p^{*}$.

Now, we define the generalized Bernoulli numbers of higher order with $\chi$ as

$$
B_{n, \chi}^{(m)}=\int_{X} \cdots \int_{X} \chi\left(x_{1}+\cdots+x_{m}\right)\left(x_{1}+\cdots+x_{m}\right)^{n} d \mu_{1}\left(x_{1}\right) \cdots d \mu_{1}\left(x_{m}\right) .
$$

We easily get in (2.12)

$$
B_{n, \chi}^{(m)}=l^{n-m} \sum_{x_{1}, \ldots, x_{m}=0}^{l-1} B_{n}^{(m)}\left(\frac{x_{1}+\cdots+x_{m}}{l}\right) \chi\left(x_{1}+\cdots+x_{m}\right),
$$

where $B_{n, \chi}$ is the generalized ordinary Bernoulli number with $\chi$.

By (2.12), we have

$$
B_{n, \chi}^{(m)}=\lim _{\rho \rightarrow \infty} \frac{1}{\left(\bar{f} p^{\rho}\right)^{m}} \sum_{1 \leq x_{1} \leq \bar{f} p^{\rho}} \cdots \sum_{1 \leq x_{m} \leq \bar{f} p^{\rho}} \chi\left(x_{1}+\cdots+x_{m}\right)\left(x_{1}+\cdots+x_{m}\right)^{n} .
$$

The investigation of these numbers is left to the interested reader.

\section{Acknowledgment}

This paper was supported by Korea Research Foundation Grant (KRF-2003-05-C00009).

\section{References}

[1] L. Carlitz, q-Bernoulli numbers and polynomials, Duke Mathematical Journal 15 (1948), 9871000.

[2] I.-C. Huang and S.-Y. Huang, Bernoulli numbers and polynomials via residues, Journal of Number Theory 76 (1999), no. 2, 178-193. 
[3] T. Kim, On a q-analogue of the p-adic log gamma functions and related integrals, Journal of Number Theory 76 (1999), no. 2, 320-329.

[4]_ q-Volkenborn integration, Russian Journal of Mathematical Physics 9 (2002), no. 3, 288 298.

[5] _ An invariant p-adic integral associated with Daehee numbers, Integral Transforms and Special Functions 13 (2002), no. 1, 65-69.

[6] _ On p-adic q-L-functions and sums of powers, Discrete Mathematics 252 (2002), no. 1-3, 179-187.

[7] __ Non-Archimedean q-integrals associated with multiple Changhee q-Bernoulli polynomials, Russian Journal of Mathematical Physics 10 (2003), no. 1, 91-98.

[8] _ On Euler-Barnes' multiple zeta functions, Russian Journal of Mathematical Physics 10 (2003), no. 3, 261-267.

[9] _ _ p-adic q-integrals associated with the Changhee-Barnes' q-Bernoulli polynomials, Integral Transforms and Special Functions 15 (2004), no. 5, 415-420.

[10] Analytic continuation of multiple q-zeta functions and their values at negative integers, Russian Journal of Mathematical Physics 11 (2004), no. 1, 71-76.

[11] T. Kim and S. H. Rim, On Changhee-Barnes' q-Euler numbers and polynomials, Advanced Studies in Contemporary Mathematics 9 (2004), no. 2, 81-86.

Lee-Chae Jang: Department of Mathematics and Computer Science, KonKuk University,

Chungju 380-701, South Korea

E-mail address: leechae-jang@hanmail.net

Seoung-Dong Kim: Department of Mathematics Education, Kongju National University, Kongju 314-701, South Korea

E-mail address: sdkim@kongju.ac.kr

Dal-Won Park: Department of Mathematics Education, Kongju National University, Kongju 314-701, South Korea

E-mail address: ysro@kongju.ac.kr

Young-Soon Ro: Department of Mathematics Education, Kongju National University, Kongju 314-701, South Korea

E-mail address: dwpark@kongju.ac.kr 